\title{
Scanning surface-enhanced Raman spectroscopy of silver nanowires
}

\author{
Stergios J. Papadakis ${ }^{1}$, Joseph A. Miragliotta ${ }^{1}$, Zhiyong Gu${ }^{2}$, David H. Gracias ${ }^{2}$ \\ ${ }^{1}$ Johns Hopkins University Applied Physics Laboratory, 11100 Johns Hopkins Rd., \\ Laurel, MD, USA 20723-6099. \\ ${ }^{2}$ Department of Chemical and Biomolecular Engineering, \\ ${ }^{3}$ Department of Chemistry, Johns Hopkins University, 3400 North Charles St., \\ Baltimore, MD, USA 21218.
}

\begin{abstract}
We report results of scanning micro-Raman spectroscopy obtained on isolated nanowires and networks of nanowires with different geometries and surface morphologies. We measured a strong, relatively homogeneous, surface enhancement of the Raman response from nanowires with a rough surface morphology, and detected a more sporadic enhanced response detected from smooth nanowires. These results provide the first steps towards the development of selective sensors for hazardous bio- and chemical-agent detection that rely on a combination of electronic conductance measurements and Raman spectroscopic measurements from metallic nanowire networks.
\end{abstract}

Keywords: SERS, sensors, nanofabrication, bio-agent detection

\section{INTRODUCTION}

Nanoparticles have been demonstrated to act as very sensitive chemical and biological sensors [1-6]. Typically, this high sensitivity is due to their very large surface area-to-volume ratio, so analytes adsorbing on their surfaces dramatically alter their properties. However, nanoscale sensors are often very sensitive to a broad range of analytes, and may therefore be of limited utility in applications such as hazardous chemical detection in realistic environments. One major challenge in creating a sensor system that is effective in real-world situations is enhancing the selectivity of such sensors. Some strategies to enhance selectivity are to design the selectivity into the sensor itself, for example by chemical functionalization of the surface which limits interaction to a narrow class of analytes; or to add filters to the front end which minimize transmission of analytes not of concern. We are pursuing a sensor system which will apply a range of orthogonal spectroscopic sensing techniques to the problem of detecting chemical agents and biomarkers in complex media. Spectroscopic techniques are promising for samples taken from complex realistic environments because they provide "fingerprints" of the analytes present, unlike simpler techniques which may only provide a change in a parameter, for example conductivity or capacitance. This also allows one sensor to detect many different analytes, rather than requiring separate selective sensors for each compound of interest. Orthogonal techniques each provide a data set that does not overlap those of the other sensing techniques in the sensor system, increasing the available information about the analytes present.

Another challenge in creating sensor systems for complex environments is that of sample collection and processing. A sensor system that has at its core a nanoscale sensor can only sample a nanoscale volume at any given time. For example, a single molecule of analyte in a cube one micron on a side (one femtoliter) is approximately one nanomolar concentration. Many of the hazardous analytes one needs to detect are dangerous at lower concentrations than this. Hence it is necessary to incorporate some method of concentrating the analytes of interest from a large, complex sample into the sensor system.

Plasmonics: Metallic Nanostructures and Their Optical Properties III, edited by Mark I. Stockman, Proceedings of SPIE Vol. 5927 (SPIE, Bellingham, WA, 2005) - 0277-786X/05/\$15 · doi: 10.1117/12.617885 
In this study, we report on progress towards a metallic nanowire network-based sensor element which combines enhanced Raman spectroscopic measurements, resulting from the surface-enhancing properties of the nanowires, with electronic tunneling spectroscopic measurements through the network. The concept is straightforward: a network of metallic nanowires is fabricated between two electrodes. There are a small number, of order 10 to 100 , junctions between nanowires in the network. The electronic transport through the network is dominated by electronic tunneling through those junctions [7-9]. When an analyte adsorbs or bonds to the surfaces of the nanowires, it is expected to change the tunneling properties of the junctions. Detection events will be transduced through a tunneling spectroscopic measurement and, simultaneously and independently, a Raman spectroscopic measurement of the analytes adsorbed on the network surface. By considering the sensor element as a component of a complete sensor system including collection, processing, and sensing elements, some advantages of this tunneling- and Raman-based sensing technique are clear: it does not require the addition of reagents or chemical markers. Therefore the sample can be passed through this measurement stage without affecting the ability of downstream sensor stages to perform their functions.

Nanowires are utilized for the core of this sensor element because of their geometry, which allows for the fabrication of a network with a relatively small number of junctions but that still covers a reasonable area on the substrate. It is well-known that agglomerated silver nanoparticles with diameters of roughly $20 \mathrm{~nm}$ strongly enhance the Raman response of molecules adsorbed to their surfaces [10]. However, this enhancement is strongest if the analyte is adsorbed to the nanoparticle surfaces prior to agglomeration. Furthermore, it is not possible to assemble a network of 20-nm spherical particles with a small number of junctions between two electrodes fabricated by simple optical lithography, because they will have a gap of at least one micron. A tunneling spectroscopic measurement through such a dense agglomeration of nanoparticles with millions of junctions, where only those at the surface are likely to be affected by the adsorption of analytes, is likely to have much lower sensitivity to the analyte than a measurement on a sparse nanowire network with few junctions.

With this sensor-element geometry in mind, we used scanning micro-Raman spectroscopy to measure the surface-enhanced Raman spectrum of silver nanowires sparsely distributed on a silicon substrate and exposed to dipicolinic acid, a common bio-marker useful in detecting the presence of certain bacterial spores. The results will be used to inform the nanowire fabrication and the network assembly, in order to optimize the Raman response of analytes adsorbed on the network.

\section{FABRICATION}

\section{a. Nanowire fabrication}

The silver nanowires were fabricated by templated electrodeposition [11, 12] using track-etched polycarbonate templates with nominal pore diameters of $30 \mathrm{~nm}$ and $50 \mathrm{~nm}$. Commercially-available tracketched membranes (created by damaging a polymer film with nuclear reaction products and then etching the damaged regions) were used. One side of the membrane was coated with a silver seed layer using thermal evaporation. Using a commercial silver plating solution, silver was electrodeposited into the pores. The membrane was dissolved in methylene chloride, freeing the nanowires from the template. The methylene chloride was then replaced with ethanol, using repeated centrifugation and sonication cycles, and the wires were stored in ethanol. Throughout this paper, we use the nominal pore size to identify the wires. The actual diameters typically were significantly larger than this (Fig. 1), with a taper at one end where the wire was deposited near the filter's front face. The vast majority of the wires were of the form shown in panels $a-b$, with relatively smooth surfaces. A small number of $50 \mathrm{~nm}$ wires were found with a much rougher surface, due to template inhomogeneity, as in panel c. As described below, these were particularly Raman-enhancing, and well-suited to sensor applications. 

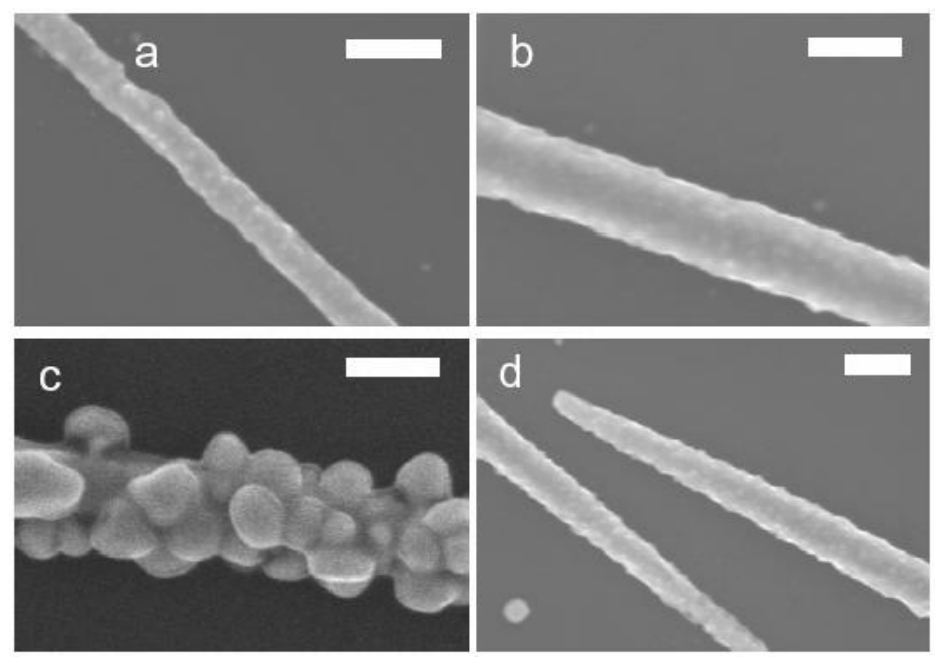

Figure 1: SEM images of the nanowires. The scale bar in each image is $200 \mathrm{~nm}$. a) Nominally $30-\mathrm{nm}$ wires. The typical actual diamater is $\sim 50 \mathrm{~nm}$. b) Nominally $50-\mathrm{nm}$ wires. The typical actual diameter is $\sim 130 \mathrm{~nm}$. c) Rough $50 \mathrm{~nm}$ wires. d) Wires showing the taper at one end.

\section{b. Dielectrophoretic network assembly}

Dielectrophoresis was used to assemble the nanowire networks. Dielectrophoresis involves the manipulation of matter using electric fields $[13,14]$. A charge-neutral object that has a dipole moment, when placed in an electric field gradient, will be acted on by a net force in the direction of the gradient (Fig. $2 \mathrm{a}$ and $2 \mathrm{~b}$ ). Dielectrophoresis combines this effect with the AC-polarizability of matter. An object, when placed in an oscillating electric field, acquires an oscillating polarization whose magnitude and phase are a function of the object's dielectric properties, conductivity, and the magnitude and frequency of the electric field. If the electric field has a spatial gradient, the object will be attracted to or repelled from the direction of increasing field depending on the relative phase of the induced dipole moment and the electric field. In practice, nanowires are suspended in a liquid, which also is polarizable, so the force is determined by the relative polarizabilities of the nanowire and the liquid. The network shown in Fig. 2c was assembled from silver nanowires using a sinusoidal peak-peak voltage between the electrodes of $0.4 \mathrm{~V}$ at a frequency of $100 \mathrm{kHz}$. The total time required to form the network was 10 seconds. This demonstrates that nanowire networks can be formed in a straightforward manner. Using dielectrophoresis, networks can be formed between many electrodes simultaneously, so the technique is scalable to the wafer level. 

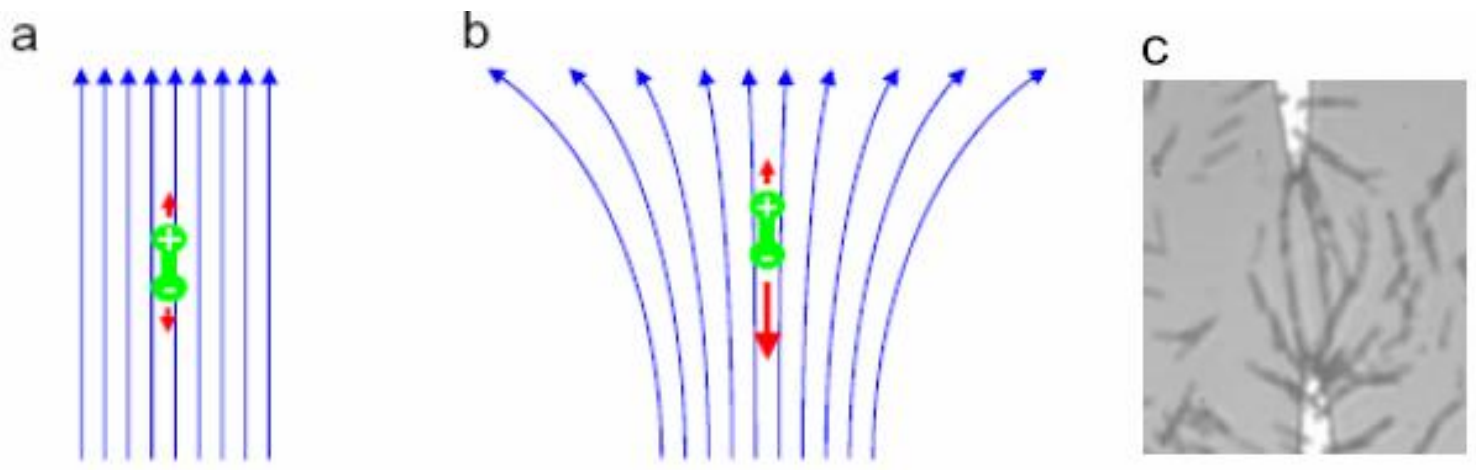

Figure 2: a) A dipole in a uniform electric field, experiencing no net force. b) A dipole in a nonuniform field, experiencing a net force in the direction of the gradient. c) A silver nanowire network formed between two electrodes. The gap between the electrodes is $\sim 20 \mu \mathrm{m}$.

\section{SCANNING SERS MEASUREMENTS:}

Measurements were performed in a scanning confocal Raman spectrometer (Fig. 3). A $514 \mathrm{~nm}$ argon laser source, conditioned by focusing through a $100 \mu \mathrm{m}$ aperture and passing through an interference filter, was focused on the sample through a 50X objective with a numerical aperture of 0.8. A $514 \mathrm{~nm}$ holographic notch filter was used to reduce the laser-wavelength light reaching the spectrometer. Spectra were recorded with a thermoelectrically-cooled charge-coupled device (CCD) detector. The sample was mounted on a computer-controlled scanning stage, which was rastered under the objective, and a complete spectrum was recorded for each pixel in the scan. All data shown in this report were acquired with a laser power, measured at the back aperture of the objective, of $75 \mu \mathrm{W}$ and an integration time for each pixel of 0.5 seconds. Calibration was done with a bulk sample of chloroform, which provides a well-known Raman spectrum. 


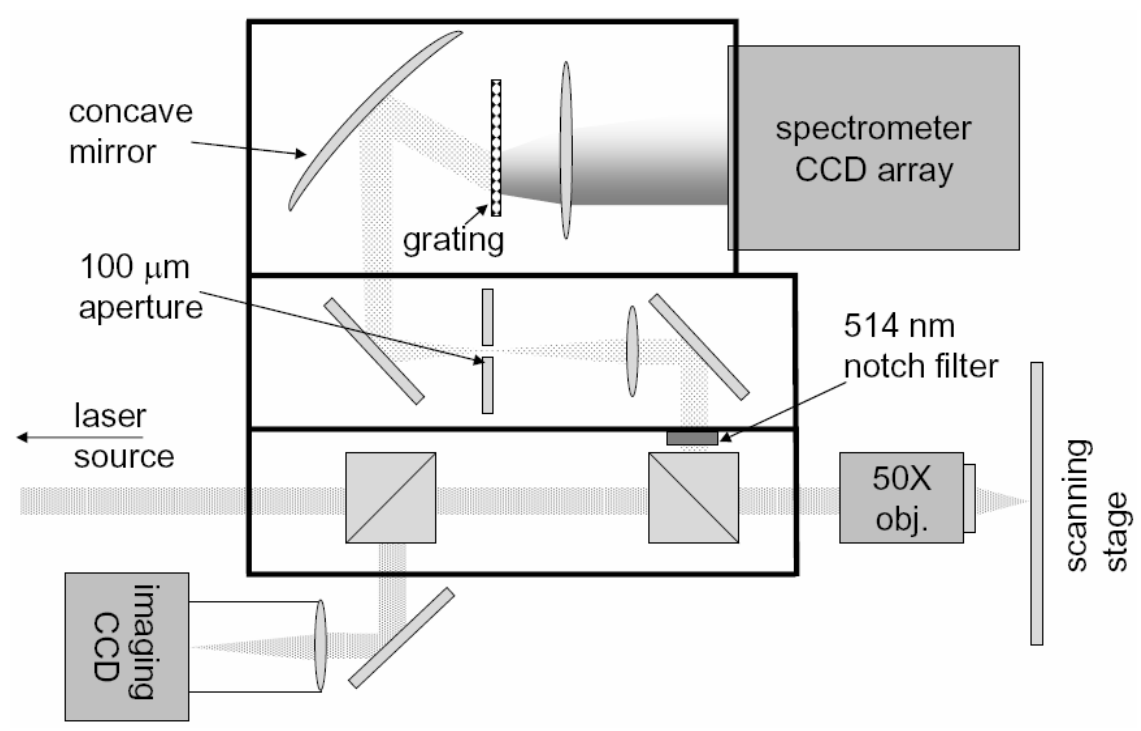

Figure 3: Schematic of the scanning Raman spectrometer. It is housed in an enclosure represented by the black lines. The convoluted path from the sample to the spectrometer, through multiple separations within the enclosure, minimizes stray light reaching the spectrometer.

In our first studies, samples with randomly distributed wires of both nominal diameters $(30 \mathrm{~nm}$ and $50 \mathrm{~nm})$ were scanned. The nanowire samples were created by casting a nanowire suspension onto a clean silicon substrate. This method produced networks of nanowires that were similar to what could be produced by dielectrophoresis, although not between two chosen electrodes. The ease of fabrication allowed a larger number of nanowire configurations to be studied quickly. The sample was then soaked in a $\sim 1 \mathrm{mM}$ dipicolinic acid (DPA) solution overnight, and then dried with a nitrogen flow, before investigation with the scanning micro-Raman spectrometer. Scanning electron microscope images of the scanned regions were also obtained, in order to be able to correlate the Raman response with particular locations on the sample.

Raman spectroscopic results from the $30 \mathrm{~nm}$ wires are shown in Fig. 4. The smooth $50 \mathrm{~nm}$ wires produced similar results. The maps in panels a-c are intensity maps of particular Raman lines from the spectra (0 $\mathrm{cm}^{-1}, 520 \mathrm{~cm}^{-1}$, and $1004 \mathrm{~cm}^{-1}$; corresponding to Rayleigh scattered light, the bulk silicon vibrational mode, and the ring-breathing mode of DPA, respectively). Panel d shows, for comparison, an image of the same region taken with a scanning electron microscope (SEM). These maps and image are representative of the results from many such measurements. The response of the nanowires, as one would expect given their large aspect ratio, has a dependence on the polarization of the incident laser light, which is horizontal in these maps. Panel a shows that the intensity of the Rayleigh scattered light is reduced when it interacts with wires oriented vertically in the image, which appear dark. This is consistent with what is seen in white-light images (not shown), where wires aligned with the polarization appear brighter, and wires perpendicular to the polarization appear darker, than the background. In contrast to this, panel $b$ shows that the intensity of the Raman response from the bulk silicon substrate is reduced by the horizontally-oriented wires. These are both expected, as light should be more strongly reflected when its polarization is aligned with the dimension of the wire that is much longer than one wavelength. When the wire is perpendicular to the polarization, a relatively greater fraction of the light is scattered away and does not reach the detector. Panel e is a background measurement; it is a typical spectrum from the silicon substrate, away from any wires. The wires produce an enhanced Raman response under some circumstances, as shown in panels f-i. The spectrum in panel $f$ is from wires just enhancing enough to be detectable above our noise floor. It is taken from point 1, and is a typical response from wires that, in SEM images, appear clean (they likely have a monolayer of adsorbed DPA or other molecules). Wires oriented perpendicular to the laser polarization usually produce such a spectrum, and can be seen in the maps of the Raman intensity with an 
appropriate choice of scale. Rarely, a wire that appears clean will produce a much more enhanced spectrum like that in panel g, from point 2. Surprisingly, most often the Raman-enhancing points are those like point 3 , where some piece of debris or crystallized organic molecules is attached to a nanowire. From high-magnification SEM images, it appears from the contrast that these objects are not metallic. It is likely that they are flakes of crystallized DPA. The three white arrows in panels $\mathrm{c}$ and $\mathrm{d}$ point to such enhancing locations. There is no enhancement from such flakes that are not attached to nanowires.

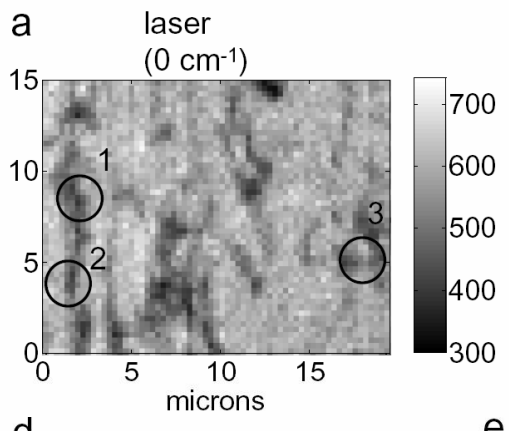

d
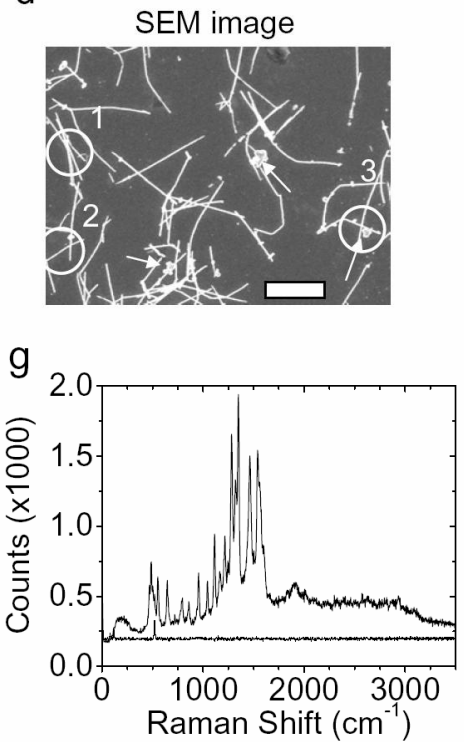

b silicon

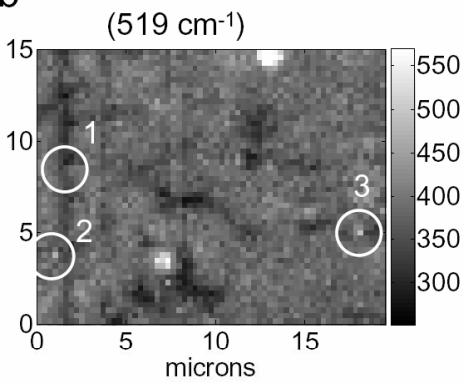

e
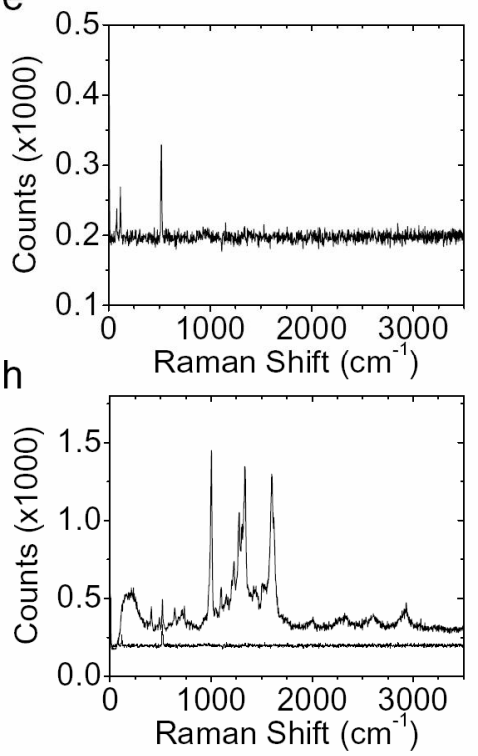

C ring-breathing mode $\left(1004 \mathrm{~cm}^{-1}\right)$

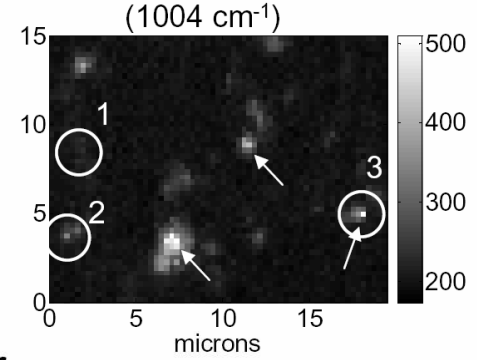

f
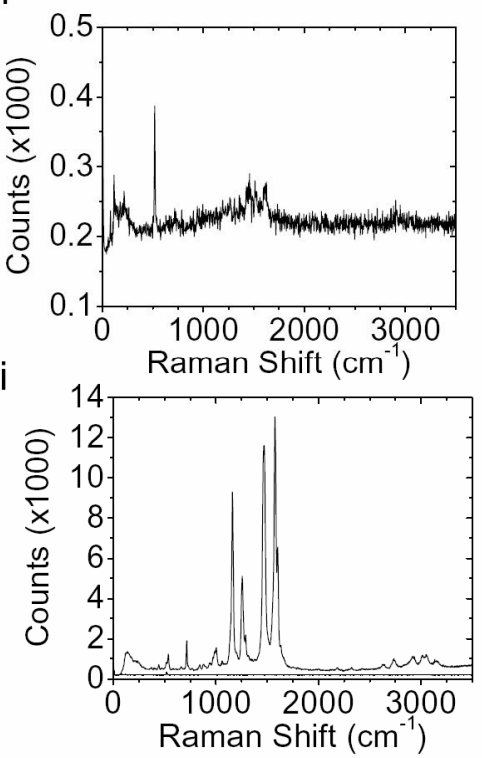

Figure 4: a) A map of the intensity of reflected laser light. b) A map of the intensity of the Raman response of the silicon substrate. c) A map of the intensity of the Raman response corresponding to the ring-breathing mode of DPA. Each pixel of the maps is $0.3 \mu \mathrm{m}$ on a side. All three maps are from the same scan, taken with the laser polarization horizontal. d) An SEM image of the same region shown in panels a-c. The white scale bar corresponds to $3 \mu \mathrm{m}$. e) A spectrum at a point on the sample away from any nanowires or features. This spectrum is also displayed in panels g-i for comparison with the signal from the nanowires. f) and g) Spectra from positions 1 and 2, respectively. h) and i) Spectra from position 3 (see text).

Examination of the spectra reveals features that are typical of very highly Raman-enhancing substrates. The ring-breathing mode response of DPA is typically seen at $\sim 1000 \mathrm{~cm}^{-1}$, although in some spectra it is very weak. Other peaks, especially in the region from $1100-1700 \mathrm{~cm}^{-1}$, where C-C stretch modes within the ring typically appear, are present in only in some spectra and not in others. It is instructive to compare spectra from different scans of the same point on the sample, as in Fig 4, panels $\mathrm{h}$ and i. These two spectra show similar signal magnitude in the ring-breathing-mode peak at $1004 \mathrm{~cm}^{-1}$ (note the different scales), but different peak positions and drastically different magnitudes in the $1100-1700 \mathrm{~cm}^{-1}$ range. Such changes in the Raman response from a given location are typical when single-molecule responses are measured [15, 
16], which strongly suggests that nanowires possess enhancement which is sufficient to produce a detectable response from a single molecule or small number of molecules. Also seen in most spectra is a peak at $\sim 2935 \mathrm{~cm}^{-1}$, which is an asymmetric C-H stretch mode typically associated with an aliphatic, not an aromatic, organic compound. Missing in these spectra, but not in the spectra from the rough wires as described below, is a peak somewhat above $3000 \mathrm{~cm}^{-1}$, which corresponds to the aromatic asymmetric $\mathrm{C}-\mathrm{H}$ stretch mode.

Raman data from the rough $50 \mathrm{~nm}$ nanowires are shown in Fig. 5. Comparing panels a and b, it is clear that the rough wires are much more Raman enhancing than the smooth wires. Both types of nanowires are present in the scanned region, but only the rough wires appear in the intensity map of the aromatic ringbreathing mode. Particularly illustrative of this response is a short smooth section of one wire which is not enhancing (indicated by the white arrow). Also, as might be expected if it is the asperities on the surface that are the central to the enhancement, there is no obvious polarization dependence of the enhancement due to the rough wires. The individual spectra, taken from various points within the network, are also more repeatable than those from the more rare Raman enhancing points found in the smooth-wire networks. The peaks corresponding to a Raman response from the DPA, the ring-breathing mode at $1004 \mathrm{~cm}^{-1}$ and the aromatic asymmetric C-H stretch at $3055 \mathrm{~cm}^{-1}$, are found in all of the spectra. The peaks at $1004 \mathrm{~cm}^{-1}$ and $3055 \mathrm{~cm}^{-1}$ are similar in magnitude from one location to the next, as is the peak at $1570 \mathrm{~cm}^{-1}$, which is likely from a $\mathrm{C}-\mathrm{C}$ stretch within the ring, although various other peaks change position and intensity, again implying that either single molecules or a very small number of molecules are being interrogated. Again, some other peaks indicate that an aliphatic contaminant is also attached to the wires, for example the $\mathrm{C}-\mathrm{H}$ stretch at $2935 \mathrm{~cm}^{-1}$.

Since the nanowires have been exposed to quite a few different compounds from their growth through to sample fabrication, and our measurements are done in the ambient environment, we cannot be certain that only DPA is on the surfaces of the wires. This makes the determination of the origins of some of the peaks speculative, especially those from the smooth wires as depicted in Fig. 4. However, the fluctuations in intensity of some of the peaks from one scan to the next, and spatially from one pixel of a scan to the next, most likely arise from effects such as spectral diffusion or from differences in the details of the binding of the molecules to the silver wires which may affect their vibrational modes $[15,16]$. However, we cannot rule out the possibility that other compounds are also bound to the wires and may contribute to the complexity of the Raman scattering response. In either case, the sudden changes in intensity from pixel to pixel and from scan to scan imply that the nanowires are enhancing enough that only a small number of molecules are responsible for the spectra observed. From the rough wires summarized in Fig. 5, similar results are obtained. In these samples, the repeatability of the spectra from point to point is sufficient for us to conclude that we have two main species on the wire surface, an aromatic compound that is most likely the DPA, and an aliphatic compound that probably results from some part of the processing.

a

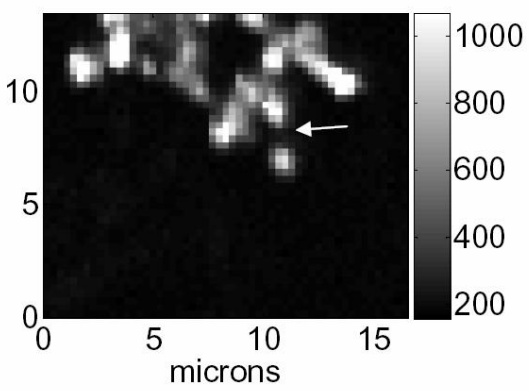

b

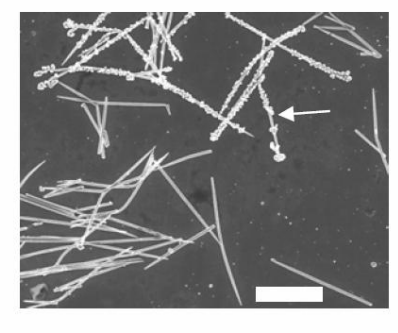

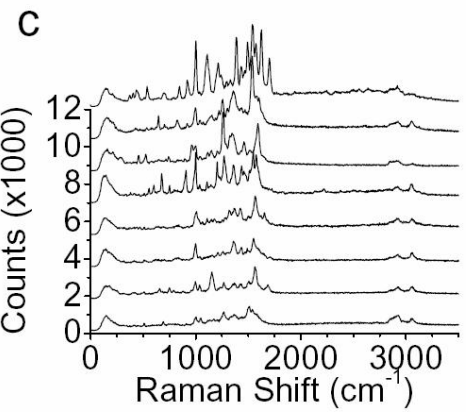

Figure 5: a) A map of the intensity of the ring breathing mode $\left(1004 \mathrm{~cm}^{-1}\right)$. Each pixel is $0.3 \mu \mathrm{m}$ on a side. b) An SEM image of the same region. The scale bar is $3 \mu \mathrm{m}$ long. c) Raman spectra taken from various points on the rough nanowires. 


\section{CONCLUSIONS:}

In summary, with the goal of investigating the utility of a nanowire network composed of a small number of nanowires (of order 10-100) as a sensor core that would use both electronic tunneling spectroscopy and optical vibration spectroscopy as transduction techniques, we have investigated the Raman surfaceenhancing properties of individual and small networks of silver nanowires. We have investigated nanowires created from $30-\mathrm{nm}$ templates and $50-\mathrm{nm}$ templates, which produced wires with actual diameters of typically $50 \mathrm{~nm}$ and $130 \mathrm{~nm}$ respectively. We also discovered that some nominally $50-\mathrm{nm}$ wires were produced that had rough surfaces, with asperities a few 10s of nanometers in diameter. All of the wires produced a strong enhancement of the Raman response of organic molecules adsorbed on their surfaces at some locations within the networks, hinting at the ability to do single-molecule vibrational spectroscopy. However, the most enhancing locations in the smooth-wire networks seemed to be associated both with nanowires and other clumps of either debris or crystallized organic material (likely dipicolinic acid), and were quite sparse. This creates a challenge for the repeatable fabrication of such enhancing locations. The rough nanowires demonstrated large Raman enhancement everywhere along their lengths, providing a nearly optimum solution for the goal of a combined tunneling spectroscopic/Raman spectroscopic sensor: a small network of such wires can be created with few junctions, which dominate the electronic transport behavior of the system, while the entire surface of the network acts as a Raman-enhancing surface for the vibrational spectroscopic measurement. Furthermore, the Raman spectra were repeatable enough within the network and had high enough fidelity that we can conclude that the wires had adsorbed not only the dipicolinic acid that we had intentionally exposed them to, but also another aliphatic compound.

\section{REFERENCES:}

1. Cui, Y., et al., Nanowire Nanosensors for Highly Sensitive and Selective Detection of Biological and Chemical Species. Science, 2001. 293: p. 1289.

2. Kolmakov, A., et al., Detection of $\mathrm{CO}_{2}$ and $\mathrm{O}_{2}$ Using Tin Oxide Nanowire Sensors. Adv. Mat., 2003. 15(12): p. 997.

3. $\quad$ Kong, J., et al., Nanotube Molecular Wires as Chemical Sensors. Science, 2000. 283: p. 622.

4. Yun, M., et al., Nanowire growth for sensor arrays. Proc. SPIE - Nanofabrication Technologies, 2003. 5220: p. 37-45.

5. Zhang, D., et al., Detection of $\mathrm{NO}_{2}$ down to ppb Levels Using Individual and Multiple $\mathrm{In}_{2} \mathrm{O}_{3}$ Nanowire Devices. Nano Lett., 2004. 4(10): p. 1919-1924.

6. Wan, Q., et al., Fabrication and ethanol sensing characteristics of $\mathrm{ZnO}$ nanowire gas sensors. Appl. Phys. Lett., 2004. 84(18): p. 3654.

7. Parthasarathy, R., X.-M. Lin, and H.M. Jaeger, Electronic Transport in Metal Nanocrystal Arrays: The Effect of Structural Disorder on Scaling Behavior. Phys. Rev. Lett., 2001. 87(18): p. 186807.

8. Ancona, M.G., et al., Coulomb blockade in single-layer Au nanocluster films. Phys. Rev. B, 2000. 64: p. 033408 .

9. $\quad$ Muller, K.-H., et al., Percolation model for electron conduction in films of metal nanoparticles linked by organic molecules. Phys. Rev. B, 2002. 66: p. 075417.

10. Moskovits, M., Surface-enhanced spectroscopy. Rev. Mod. Phys., 1985. 57(3): p. 783.

11. Martin, C.R., Nanomaterials: A Membrane-Based Synthetic Approach. Science, 1994. 266: p. 1961.

12. Huczko, A., Template-based synthesis of nanomaterials. Appl. Phys. A, 2000. 70: p. 365.

13. Jones, T.B., Electromechanics of Particles. 1995, Cambridge, New York: Cambridge University Press.

14. Pohl, H.A., Dielectrophoresis : the behavior of neutral matter in nonuniform electric fields. 1978, Cambridge, New York: Cambridge University Press.

15. Meixner, A.J., T. Vosgr, and M. Sackrow, Nanoscale surface-enhanced resonance Raman scattering spectroscopy of single molecules on isolated silver clusters. J. Lumin., 2001. 94-95: p. 147.

16. Michaels, A.M., M. Nirmal, and L.E. Brus, Surface Enhanced Raman Spectroscopy of Individual Rhodamine 6G Molecules on Large Ag Nanocrystals. J. Am. Chem. Soc., 1999. 121: p. 9932. 